\title{
Le voyage révélateur dans la littérature de l'immigration de deuxième génération. Le cas des allers-retours interculturels d'écrivains belges d'origine marocaine et turque ${ }^{1}$
}

\section{José Domingues de Almeida}

Universidade do Porto - ILC

Résumé: Cet article tente d'approcher la thématique de la mobilité tant géographique que culturelle de quatre auteurs belges francophones issus, à différents égards, de l'immigration et dont le statut migrant engage une réflexion sur le vécu des deuxième et troisième générations d'immigrés, et leur rapport à la culture du pays d'origine. La notion même de "littérature migrante" se voit dès lors convoquée et illustrée à la faveur du vécu interculturel.

Mots-clés: littérature migrante, aller-retour, interculturalité, Belgique, immigration

Resumo: Este artigo propõe-se abordar a temática da mobilidade tanto geográfica como cultural de quatro escritores belgas de língua francesa oriundos, a vários títulos e níveis, da imigração, cujo estatuto migrante (literatura migrante) implica uma reflexão sobre a experiência das segunda e terceira gerações de imigrados, e sobre a sua relação com a cultura do país de origem. A própria noção de "literatura migrante" vê-se assim convocada e ilustrada pela própria vivência intercultural.

Palavras-chave: literatura migrante, ida e volta, interculturalidade, Bélgica, imigração 
Dans le cadre contemporain des lettres belges, les auteurs retenus ici (Malika Madi, Altay Manço, Kenan Görgun et Mina Oualdlhadj) ressortissent à la génération d'écrivains qui émerge dans la foulée du débat identitaire et culturel de la belgitude, qui en a profité, mais l'a assurément oublié, et dont la référence au lieu d'édition et l'inscription géographique de la diégèse indiffèrent, même s'ils sont bien nés quelque part, et que cet ici décomplexé est nettement reconnaissable (Lorent 1997: 52-62).

Mais force est de constater la spécificité identitaire d'une fiction narrative suscitée par le vécu migrant, laquelle interroge, tout en les dépassant, les littératures nationales, et projette les littératures francophones dans une dimension mondialisée, mais toujours en quête de légitimité institutionnelle et de stabilité taxinomique.

Ces écrivains se trouvent dans des circonstances fort distinctes de celles qui prévalurent lors de l'émergence des premiers écrivains maghrébins de la toute première génération immigrée et dont, selon Charles Bonn, "la fonction [était] de faire exister (...) l'espace identitaire par affirmation à la face du monde" (Bonn 2012: 12). Elle devait culminer et se faire reconnaître par les instances de légitimité française, entre autres, avec Tahar Ben Jelloun. Dans la plupart des cas, il s'agissait d'évoquer la "catastrophe" et le "sacrifice" impliqués dans le fait migratoire.

L'écriture fictionnelle de ces écrivains (deuxième, voire troisième génération) relève d'un tout autre projet, plus complexe et plus difficilement classable. Plusieurs taxinomies (littérature beure, migrante, de l'immigration, etc.) s'en sont emparées sans pour autant épuiser la totalité des questions identitaires et critiques soulevées. Il s'agit en fait d'une écriture défiant le statut académique des littératures nationales et qui, dès lors, s'impose par l'innovation des enjeux thématiques et narratifs.

Étant donné le caractère récent du phénomène, sans recul historique (première lacune relevée par Elien Declercq (2011: 306)), la critique n'en a pour l'heure qu'une perception micro-diachronique, pour reprendre Michel Laronde (1993: 221), ce qui en fait un corpus instable et hybride (idem: 222). Jean-Marc Moura le définit comme de "nouveaux territoires" narratifs fondés sur l'émergence de nouvelles communautés sociales, constituant un objet d'étude complexe, surtout à partir des années 1990, où la 
question du statut des générations immigrées, et de leurs conflits identitaires au sein de la Nation est venue se poser de façon plus aigüe (Moura 2003: 57).

La littérature nationale aura du mal à intégrer ces littératures migrantes dans ses définitions taxinomiques. Elien Declercq insiste sur le "nouveau dialogue" qu'elles établissent avec le concept de littérature nationale dans la mesure où elles en interrogent les contours tout en convoquant dans le débat des "échanges discursifs" au-delà des thématiques habituellement soulevées par l'étude des flux migratoires dans leur acception économique et sociale (Declercq 2011: 301).

Aussi l'approche métaphorique avancée par Habiba Sebkhi s'avère-t-elle stimulante en vue d'une distinction de nature juridique entre une littérature légitime contrôlée, et une autre, illégitime, naturelle, et dès lors bâtarde, ou en tous cas "hybride" (Sebkhi 1999). Comme le rappelle Hans-Jürgen Lüsebrink, cette hybridité est le fait des phénomènes de métissage et de créolisation opérés, en contexte globalisé, par les flux migratoires; lesquels débouchent sur une "poétique interculturelle" (Lüsebrink 2003: 64-65) qui pourrait nous réserver encore bien des surprises.

D'ailleurs, rappelons que la Belgique littéraire, notamment dans le, voire grâce au discours de la belgitude, échappe précisément au fait des littératures nationales et à l'hypostase nationale hexagonale. Elle s'avère donc, comme l'ont en tous les cas affirmé ses acteurs, une terre où le va-et-vient culturel et intellectuel, ainsi que l'exil intérieur sont constitutifs, la bâtardise étant explicitement revendiquée dans les manifestes de la belgitude, entre autres topoï identitaires et littéraires.

En fait, dans le contexte des études francophones, les littératures migrantes complexifient les données. Si Guy Dugas voit déjà dans la francophonie littéraire "une notion mouvante” et “non-stabilisée”” (Dugas 1992: 16), Anne-Rosine Delbart voit, elle, dans les productions littéraires beures une littérature:

(...) $\min$ [ant] de l'intérieur le concept de littérature nationale, entendez ici littérature française, comme le mine de l'extérieur cette fois les auteurs venus d'ailleurs, tous ces auteurs non français qui prennent la plume en français et publient en France, qu'ils soient ou non de langue maternelle française et qu'on rattache au champ de la ou des littératures francophones. Alors, face à ces phénomènes littéraires 
relativement nouveaux, quelle dénomination privilégier? La récente proposition d'une littératuremonde participe explicitement de la volonté de délier le pacte langue-nation et s'affiche comme le substitut idéal à la trop contestée étiquette de littérature francophone qui "entérine une ségrégation". (Delbart 2010: 107)

Pour Pierre Halen, il s'agirait d'exclure les littératures migrantes du groupe des "convertis", qui ont fait allégeance au système littéraire francophone (Halen 2003: 33). Cette hybridité adviendrait, selon Christiane Albert, de l'introduction même du thème de l'exil dans le fait littéraire francophone (Albert 2005: 14); un topos dont l'origine n'est pas seulement coloniale, - aujourd'hui globale -, vu qu'il trouvait déjà des échos revendicatifs dans l'argumentaire de la belgitude et dans ceux qui virent le jour dans d'autres périphéries francophones, mais dont le discours fictionnel produit demeure encore inclassable en France, alors qu'il a tout naturellement et spontanément trouvé sa place dans d'autres champs littéraires et linguistiques (Pays-Bas ou Suède).

En outre, toute tentative de classement taxinomique de ces littératures se heurte à l'identité ethnique de l'auteur et / ou aux représentations relayées par le figement racial du personnage principal (idem: 18), souvent sujet d'un témoignage empirique, et ce, bien plus qu'aux critères esthétiques intrinsèque du texte. En effet, "beure", "migrante" ou écriture de l'“entre-deux" (idem: 149ss) pointent autant de descripteurs marqués par l'appartenance ethnique, raison qui explique la "francophonisation" de ces textes.

Pour prévenir cet écueil, Elien Declercq suggère la désignation "littérature de migration", une taxinomie qui a l'avantage de pallier une lacune théorique liée à un paradigme conceptuel (Declercq 2011: 308-310). D'autant plus que le phénomène migratoire s'avère plus complexe qu'on ne le pense, et peut même ne pas impliquer directement le brassage ethnique. Delbart ne signale-t-elle pas l'exemple d'un écrivain français pure souche jouant le jeu onomastique et thématique des écritures migrantes (Delbart 2010: 105)?

Illustrons donc cette fiction découlant du phénomène par la brève évocation de quatre textes écrits par quatre écrivains belges issus de l'immigration. Mina Oualdlhadj est née au Maroc en 1964. À onze ans, elle rejoint son père parti travailler en Belgique. Elle y décroche 
une licence en langue et littérature françaises, et travaille comme médiatrice scolaire et animatrice socioculturelle. Mariée, mère de trois enfants, à qui elle dédie son roman Ti t'appelles Aïcha, pas Jouzifine! (2008), elle dirige depuis 2001 plusieurs crèches communales.

Par ailleurs, d'origine turque, Kenan Görgün est né à Gand en 1977. Il a fait ses études à Bruxelles dans une école francophone, suite au déménagement de sa famille turque immigrée. À quinze ans, il décide d'écrire, suit un atelier d'écriture et contribue d'abord à la revue Marginales avant de publier ses premiers poèmes et nouvelles. Il devient scénariste et parolier pour le groupe de rock O.I.L. Il est l'auteur de plusieurs romans et essais, dont Patriot act (2009) et le récit J'habite un pays-fantôme (2014) qui nous occupera ici, et a fait l’objet de représentations.

Docteur en psychologie, directeur scientifique de l'Institut de Recherche, Formation et Action sur les Migrations (IRFAM), Altay Manço est, lui, l'auteur d'un grand nombre d'essais sur l'intégration des populations immigrées et les relations interculturelles, notamment entre la Belgique, dont il est ressortissant, et la Turquie, dont sa famille est originaire. Ses travaux académiques et son premier roman, Métissages 100\% (Manço, 2012) se penchent sur les questions d'intégration des populations immigrées en Belgique, et livrent son approche personnelle de la Turquie, balancée entre tradition et modernité. Bref, une Turquie en mouvement, même si ces voyages imaginaires sont avant tout immobiles et intérieurs.

Enfin, d'origine algérienne, Malika Madi est née en Belgique, fille d'un immigré arrivé dans ce pays en 1960 pour travailler dans la mine. Toute petite, elle se découvrit une passion pour l'écriture et la langue française. Avant d'écrire un vrai roman, elle commença par écrire des nouvelles. Diplômée en gestion, elle a publié son premier roman Nuit d'encre pour Farah (2000). Un deuxième roman, Les silences de Médéa est paru en 2007. Aujourd'hui, tout en continuant son travail d'écriture, elle dirige des ateliers d'écriture et donne des conférences dans des bibliothèques de la Communauté Française de Belgique.

Ces quatre écrivains ont ceci en commun qu'ils appartiennent à la deuxième génération immigrée en Belgique (ces Turcs ou Maghrébins partis aux Pays-Bas ou en 
Belgique, ou ces Italiens arrivés depuis des décennies, ou nés en Wallonie dont parle Elien Declercq, Nicole Malinconi, entre autres (2011: 305)) et qu'ils ont vécu l'entre-deux identitaire entre ce pays d'accueil, - dont ils ont tout naturellement acquis la nationalité -, et celui de leurs ancêtres. Aussi est-il possible, dans les quatre cas, de dégager des points de convergence thématiques et esthétiques. Et tout d'abord, le caractère autobiographique et la valeur du témoignage sur le vécu, dont parle Anne-Rosine Delbart (2010: 104-105). Mina Oualdlhadj n'entend-elle pas son projet de parole libératrice (2008: 114) en tant qu'“exutoire” (idem: 7)? D’ailleurs, le caractère autobiographique du roman est affiché d'emblée de jeu: "Depuis que mon père a pris sa retraite, mes parents vivent en Belgique, mon pays de naissance, et le Maroc, mon pays d'origine" (idem: 14).

Par ailleurs, l'entre-deux identitaire trouve chez ces quatre auteurs belges une expression particulière que Delbart a bien relevé dans la littérature migrante sous les traits d'une "interculturalité réussie" et d'un "cheminement vers l'autre" (2010: 100) qui engendrent des processus de "transculturation" et des "hiatus culturels" (idem: 101). En fait, il s'agit de traduire la double appartenance enrichissante, mais sur base d'un retour ou va-et-vient décevant au pays d'origine des parents; ce voyage devenant révélateur d'une appartenance à l'ici et à l'ailleurs (apud Delbart 2010: 103) et donnant lieu à une sorte de "négociation" (ibidem). Dans Ti t'appelles Ä̈cha, pas Jouzifine!, comme le souligne AnneRosine Delbart, l'épisode du rapport à la fête de Noël en est une illustration emblématique: “À l'âge de sept ans, je fis une autre découverte décevante: saint Nicolas et le Père Noël n'étaient pas musulmans" (Oualdlhadj 2008: 53).

Ce type de hiatus est une constante dans la littérature dite "beure", comme celle de l'auteure belge d'origine algérienne, Malika Madi où l'anecdote interculturelle est fréquente. Il suffit d'évoquer l'anecdote de la "classe de neige": “- Quelle honte ! quelle honte ! quelle honte! Une classe de neige! Mais tout le monde va nous montrer du doigt, on va être la honte du quartier. Comment oses-tu me demander une chose comme celle-là?... Des filles et des garçons mélangés !" (Madi 2013: 52); ou encore l'épisode drolatique du mouton dans Ti t'appelles Aïcha, pas Jouzifine! lorsque l'institutrice demande à la classe: “- Que peut-on 
trouver dans une cave, Mimi? - Un mouton! Rire des enfants et stupéfaction de l'institutrice (...)" (Oualdlhadj 2008: 50), ce qui éveillera des suspicions sur l'abattage clandestin.

Tant Ti t'appelles Aïcha, pas Jouzifine! que Nuit d'encre pour Farah constituent des récits de "femmes lucides" (idem: 11) foncièrement marqués par l'intransigeance paternelle à l'égard de la lutte pour l'émancipation des filles, ce qui dessine une intense négociation interculturelle et un statut d'entre-deux comme effet d'un phénomène migratoire et exilique antérieur et qui les dépasse: "Mes parents sont arrivés dans les années 60, ce temps béni où l'Europe avait encore besoin de main-d'œuvre étrangère" (idem: 39); "Et si mes parents étaient simplement des victimes de l'exil? Ne dit-on pas que l'exil provoque un renfermement sur soi?" (idem: 84). Aïcha s’affirme "coincée” (idem: 17,41), déchirée entre deux cultures où les codes culturels balisent l'existence, tels que les règles de bienséance : “Dans quel film tu as vu que j'embrasse mon géniteur sur la joue? - Pardon, je t'embrasse la tête ou la main?" (idem: 17). Dès lors, “- C'est vrai, quoi que je fasse, je suis coincée” (idem: 17).

C'est à cet égard que le voyage, - retour en zone dans le pays du père -, s'avère révélateur d'une identité inconciliable, d'une prise de conscience de la distance culturelle séparant un avant et là-bas d'un maintenant et ici belges et européens. Ti t'appelles Aïcha, pas Jouzifine! raconte les déboires de deux jeunes femmes issues de la deuxième génération immigrée en Belgique. L'une est née au Maroc, l'autre pas. L'une a des parents assez tolérants (Mimi), l'autre beaucoup moins (Aïcha). L'une a fondé sa propre famille et gère sa propre vie; l'autre pas encore. Pourtant, Aïcha et Mimi sont deux sœurs d'une même condition identitaire marquée par l'immigration en amont. Pour Aïcha, les "vacances au pays" sont l'occasion de se confronter à l'altérité chez soi. Si l'impact des douanes et des douaniers est déjà considérable (idem: 127), le plus difficile revient à gérer les décalages culturels et sociaux: "Au Maroc, l'immigré semble narguer les autres par son pouvoir d'achat. Pendant toute la durée des vacances, il ne cesse de consommer, sous les yeux de ses compatriotes. Comment voulez-vous que le Marocain moyen ne rêve pas de traverser le détroit de Gibraltar à la nage?" (idem: 130). Mais la pression est surtout mise sur l'urgence du mariage des jeunes filles. Là le choc est intense, voire fatal. Aïcha est 
immédiatement vue comme "Une jeune fille à marier" (idem: 131): "La situation devient de plus en plus critique. J'ai alors vingt-deux ans. Dans mon entourage, les filles de mon âge se marient" (idem: 134).

Même si Nuit d'encre pour Farah (2013 [2000]) n'entend pas être autobiographique, il n'est pas difficile de découvrir dans les traits des trois sœurs Farah, Lila et Latifa ceux de Malika Madi: culture et tradition musulmanes, mais option pour un style de vie totalement européen. Le voyage révélateur, en Algérie cette fois, fait apparaître des valeurs fort différentes, dont le mariage forcé. Fatah s'est en effet vue contrainte à un retour au pays pour compenser la fugue de ses deux sœurs aînées. Elle devra abandonner ses études en Belgique, elle, la dix-neuvièmiste en herbe, et mener une existence rudimentaire et traditionnelle en Algérie. Apprenant que ses sœurs ont fini par connaître un tout autre sort, qu'elles ont même obtenu le pardon paternel, et que sa vie à elle, a été sacrifiée, Fatah sombre dans la folie.

Le voyage révélateur en entre-deux, "no man's land identitaire sans ancrage national défini", dont parle Christiane Albert (2005: 21), prend chez Kenan Görgün les traits de l'indéfinition du pays-fantôme, quelque part entre Bruxelles et Istanbul (Görgün 2014: 7), “(...) une métropole qui, à elle seule, compte plus d'habitants que le pays où tu vivais hier" (idem: 42). Inscrit sous le signe exilique cher à la thématique migrante (Albert 2005: 9), ce récit glose cet indéfini comme une condition et un vécu identitaire, qui plus est dans un contexte mondialisé ou les tensions global $v s$ local sont paradoxales, voire inattendues: "La nostalgie de ses racines, dans son propre pays?", s'interroge-t-il (8), pour spécifier plus loin, en guise d'énigme: "Pays-fantôme dont il me faut sans cesse redessiner la carte. Belgique hier. Turquie aujourd'hui. Et demain, où irons-nous? De quel déracinement vers quel égarement?" (Görgün 2014: 9).

Aussi, le "village global" de Marshal McLuhan est-il confronté de façon aporistique au village natal turc des parents immigrés en Belgique: "Le monde est un village où mon village n'est plus?" (idem: 54). Ainsi la condition contemporaine globalisée fournit une métaphore transitive - "nomade moderne" (idem: 52) - à l'instabilité identitaire personnelle du narrateur: "Demain, ou après-demain, je sais que vais repartir" (idem: 52): “Encore une fois, 
plier bagages, encore, me raconter l'histoire de celui qui cherchait son identité et finirait un jour par douter même qu'il en eut jamais une (...)" (idem: 52).

Dans Métissages 100\% d'Altay Manço (2012) les négociations d'adhésion de la Turquie à l'Union Européenne servent de toile de fond à une rencontre improbable: une jeune femme issue de l'immigration turque en Belgique faisant carrière dans la mode, en conflit avec ses parents traditionnalistes, et un professeur d'histoire et de langues orientales en crise familiale, un personnage aux traits assez proches de l'auteur. Cette rencontre est l'occasion d'un voyage révélateur au centre des idiosyncrasies turques du moment, et de ses répercussions sur le vécu identitaire des Belges issus de l'immigration turque. Entre Bruxelles et Istanbul, un appel à la valeur du métissage et à la convivialité interculturelle est sans cesse repris en leitmotiv.

Le narrateur homodiégétique semble regretter le temps béni où, dans le pays de ses parents, plusieurs cultures et religions cohabitaient en paix et formaient un tout métaphorique de notre condition contemporaine. D'ailleurs, le décompte du pourcentage de métissage au cours des chapitres, pour revenir à $100 \%$ à la fin, traduit l'instabilité identitaire du narrateur. À 46\% de métissage, le narrateur avoue: "Je ne suis pas Turc. À quoi cela me mène-t-il? Comment sait-on en réalité ce que l'on est? C'est tout de même interpellant. Je suis historien pour les autres: jamais je ne me suis interrogé sur les origines de ma propre famille" (idem: 134). Ce constat en appelle un autre plus ample pour notre propos, et qui occupe d'interminables parenthèses discursives dans le roman et le fait frôler l'essai:

On constate que la majeure partie des familles de l'Asie Mineure n'a pas bougé de place depuis 40000 ans ! Il y a des migrations, mais en faible quantité. Les tests le montrent: à peine $10 \%$ à $15 \%$ des habitants de la Turquie sont biologiquement originaires de l'Asie (...). Il n'y a pas de 'gènes turcs' pas plus que de gènes d'une autre ethnie. On confond identité culturelle et héritage génétique. (idem: 135)

Ce faisant, l'auteur-narrateur renvoie dos à dos aussi bien l'idéologie d'une grande Turquie, - celle qu'il voit monter dans la politique interne turque actuelle -, que les réticences 
européennes vis-à-vis de l'adhésion de ce pays, majoritairement asiatique et musulman à l'UE.

Il appert de ce bref aperçu en survol des écrivains retenus que leur statut identitaire implique la constante de l'épreuve d'un voyage révélateur qui rappelle l'avant et l'ailleurs des parents, et les confrontent à leur ici et maintenant d'écrivains belges, de sorte que le passé des parents, de la première génération immigrée, continue d'influer sur l'existence des deuxième et troisième générations prétendument "intégrées", mais qui n’en finissent pas de se sentir en marge de la nationalité, voire du système littéraire en langue française, d'autant plus que sont apparus entretemps de nouveaux facteurs culturels prégnants, mais déstabilisateurs: la crise des foulards, l'intégrisme islamique, les flux migratoires avec leur cortège de naufrages et de déplacements.

Dans un cadre plus vaste, qui interroge l'agencement national du fait littéraire en français, ces textes problématisent et complexifient ces données. Faut-il s'habituer à l'idée de l'étranger et du voyageur absolu? Kenan Görgün nous y invite:

Plus expressif qu'un Italien, plus gouailleur qu'un Espagnol, plus protectionniste qu'un Allemand, plus calculateur qu'un Danois, et, surprise, plus talentueux pour déjouer tous les clichés dont on pourrait l'accabler - cet étranger, cet intrus, n'est-il pas tout cela à la fois, et une essence qui s'obstine à m'échapper? (Görgün 2014: 22) 


\section{Bibliographie}

Albert, Christiane (2005), L'immigration dans le roman francophone contemporain, Paris, Karthala.

Bonn, Charles (2012), "Dire la migration et catastrophe de l'entrée en littérature" in Passages et naufrages migrants. Les fictions du détroit (A. P. Coutinho, F. Outeirinho et J. Almeida éds.), Paris, L’Harmattan, 9-20.

Declercq, Elien (2011), “Écriture migrante: réflexions sur un concept aux contours imprécis", Revue de littérature comparée, $\mathrm{n}^{\circ}$ 339, 301-310.

Delbart, Anne-Rosine (2010), “Littératures de l'immigration: un pas vers l'interculturalité?", Carnets, "Littératures nationales: suite ou fin - résistances, mutations \& lignes de fuite", no spécial printemps / été, 99-110.

Dugas, Guy (1992), "Francophonie, acculturation, littératures nationales et dominées... Retour sur quelques concepts mal définis", Convergences et divergences dans les littératures francophones, Paris, L'Harmattan, 15-21.

Görgün, Kenan (2009), Patriot act, Paris, First editions.

Görgün, Kenan (2014), J'habite un pays-fantôme, Bruxelles, Couleur livres.

Halen, Pierre (2003), Le “'système littéraire francophone': quelques réflexions complémentaires" in Les études littéraires francophones: état des lieux (L. D’Hulst et J.-M. Moura éds.), Lille, Travaux du Conseil Scientifique de l'Université Charles-de-Gaulle-Lille 3, 25-37.

Laronde, Michel (1993), Autour du roman beur. Immigration et identité, Paris, L’Harmattan. Lorent, Laure Elisabeth (1997), "Les écrivains belges sont né quelque part", La Revue Nouvelle, t. 105, mars, 20-78.

Lüsebrink, Hans-Jürgen (2003), "Transferts culturels et histoire des médias: un défi pour les études francophones" in Les études littéraires francophones: état des lieux (L. D’Hulst et 
J.-M. Moura éds.), Lille, Travaux du Conseil Scientifique de l’Université Charles-de-GaulleLille 3, 59-76.

Madi, Malika (2013) [2000], Nuit d'encre pour Farah, Mons, Éd. du Cerisier.

Manço, Altay (2012), Métissages 100\%, Paris, L’Harmattan.

Moura, Jean-Marc (2003), “Les études postcoloniales: pour une topique des études littéraires francophones" in Les études littéraires francophones: état des lieux (L. D’Hulst et J.-M. Moura éds.), Lille, Travaux du Conseil Scientifique de l’Université Charles-de-GaulleLille 3, 49-61.

Oualdlhadj, Mina (2008), Ti t’appelles Aïcha, pas Jouzifine !, Nivelles, Clepsydre.

Sebkhi, Habiba (1999), "Une littérature naturelle: le cas de la littérature 'beure'”, Itinéraires et contacts de cultures, $\mathrm{n}^{\circ} 27,16-27$ (consulté sur le site www.limag.refer.org).

José Domingues de Almeida est Maître de Conférences à la Faculté des Lettres de l'Université de Porto. Il est docteur en Littérature française et francophone contemporaine (2005) à la suite de la soutenance de sa thèse intitulée : Auteurs inavoués ; Belges inavouables. Fiction, autofiction et fiction de la Belgique dans l'œuvre romanesque de Conrad Detrez, Eugène Savitzkaya et Jean-Claude Pirotte. Une triple mitoyenneté. Ses domaines de recherche sont la littérature française et francophone contemporaine, les études francophones et la culture et pensée françaises contemporaines.

\section{NOTE}

\footnotetext{
${ }^{1}$ Cet article s'insère dans la recherche menée au sein du Programme Stratégique intégré UID/ELT/00500/2013 | POCI-01-0145-FEDER-007339
} 